\title{
Chaos Analysis and Control of Relative Rotation System with Mathieu-Duffing Oscillator
}

\author{
Yu Zhang and Longsuo Li \\ Department of Mathematics, Harbin Institute of Technology, Harbin 150001, China \\ Correspondence should be addressed to Longsuo Li; lls@hit.edu.cn
}

Received 28 February 2015; Revised 21 April 2015; Accepted 21 April 2015

Academic Editor: Yang Tang

Copyright ( 2015 Y. Zhang and L. Li. This is an open access article distributed under the Creative Commons Attribution License, which permits unrestricted use, distribution, and reproduction in any medium, provided the original work is properly cited.

\begin{abstract}
Chaos analysis and control of relative rotation nonlinear dynamic system with Mathieu-Duffing oscillator are investigated. By using Lagrange equation, the dynamics equation of relative rotation system has been established. Melnikov's method is applied to predict the chaotic behavior of this system. Moreover, the chaotic dynamical behavior can be controlled by adding the Gaussian white noise to the proposed system for the sake of changing chaos state into stable state. Through numerical calculation, the Poincaré map analysis and phase portraits are carried out to confirm main results.
\end{abstract}

\section{Introduction}

Chaos, as a kind of physical phenomenon, exists widely in various nonlinear dynamic systems [1-5]. The main ideal of chaos control approach is that one can use given unstable periodic orbits or external excitations to force chaotic system into stable system [6-9]. There are many ways to be used to suppress chaos, such as parametric driven [10-12] and time delayed approach $[13,14]$, and the Gaussian white noise as random phase control is an interesting one. The control of chaos we discuss in this paper is based on this case.

The theory of mechanics of relative rotation system was established by Carmeli in 1986 [15]; bifurcation and chaos of this system got rapid development in variety of areas. Shi et al. [16] studied the chaotic behavior and its control for a class of nonlinear dynamics equation of relative rotation system. Liu et al. [17] found stability and bifurcation for a coupled nonlinear relative rotation system with multitime delay feedbacks. Therefore, this system plays an important role in nonlinear dynamic systems.

Stochastic forces or random noise are the most frequently control strategies to be used in suppressing chaos. Ramesh and Narayanan [18] investigated the robustness in presence of uniform noise and found that the system would lose control while noise intensity was raided to a threshold level. Liu et al. [19] explored the effect of bounded noise on chaotic motion of Duffing oscillator under parametric excitation. Wu et al. [20] studied stochastic chaos and its control by the top Lyapunov exponent. Yin et al. [21] proved the peculiar solitary waves are more likely to be chaos by using the Melnikov theory and found that the system can be well controlled when the frequency of the perturbation surpasses the peculiar perturbation frequency with fixed parameters of the unperturbed system. Noise, as random phase, has been used in studying the control of chaos in the paper. So as a kind of effective method of chaos control, whether in theory or in the practical application, the noise has some of the research significance.

In this paper, we explore chaos analysis and control in relative rotation system with Mathieu-Duffing oscillator. The paper is organized as follows. The dynamics equation of relative rotation system is established in Section 2. In Section 3, the prediction of chaotic motion is given by Melnikov's method. In Section 4, the Gaussian white noise can increase or decrease the threshold values of chaos, so as to realize the control of chaos.

\section{Dynamics Equation of Relative Rotation System with Mathieu-Duffing Oscillator}

Mathieu-Duffing system is a class of typical vibration system, and researching its nonlinear dynamic characteristics shows 
to be extremely essential. Considering Mathieu-Duffing oscillator expression form as follows $[22,23]$

$$
f(t)=N_{1}(t) x+N_{2}(t) x^{3},
$$

where

$$
\begin{aligned}
N_{1}(t) & =c K(t), \\
N_{2}(t) & =b K(t), \\
K(t) & =k_{0}^{2}+k_{1} \cos \left(\omega_{1} t\right)
\end{aligned}
$$

$N_{1}(t), N_{2}(t)$ are linear torsional stiffness of the system. For two quality relative rotation systems of Mathieu-Duffing oscillator, the kinetic energy is

$$
E=\frac{1}{2} J_{1} \dot{\theta}_{1}^{2}+\frac{1}{2} J_{2} \dot{\theta}_{2}^{2}
$$

where $J_{1}, J_{2}$ are moment of inertia and $\theta_{i}, \dot{\theta}_{i}(i=1,2)$ are rotational angle and speed of rotational angle, respectively. The potential energy of system is

$$
U=\frac{1}{2} N_{1}(t) \theta^{2}+\frac{1}{2} N_{2}(t) \theta^{4}
$$

Generalized forces are

$$
\begin{aligned}
& Q_{1}=F_{1}^{1} \frac{\partial \theta_{1}}{\partial q_{1}}+F_{2}^{2} \frac{\partial \theta_{2}}{\partial q_{1}}, \\
& Q_{2}=F_{1}^{1} \frac{\partial \theta_{1}}{\partial q_{2}}+F_{2}^{2} \frac{\partial \theta_{2}}{\partial q_{2}},
\end{aligned}
$$

where

$$
\begin{aligned}
& F_{1}^{1}=T_{1}-A\left(\dot{\theta}_{1}-\dot{\theta}_{2}\right), \\
& F_{2}^{2}=T_{2}-A\left(\dot{\theta}_{2}-\dot{\theta}_{1}\right),
\end{aligned}
$$

where $T_{1}, T_{2}$ are generalized external force, $q_{1}, q_{2}$ are generalized coordinates, and $A$ is linear damping coefficient. The Lagrange equation is

$$
\frac{d}{d t} \frac{\partial E}{\partial \dot{q}_{j}}-\frac{\partial E}{\partial q_{j}}+\frac{\partial U}{\partial q_{j}} .
$$

Substituting (1)-(5) into Lagrange equation (7), we have

$$
\begin{aligned}
& J_{1} \ddot{\theta}_{1}+c K(t)\left(\theta_{1}-\theta_{2}\right)+b K(t)\left(\theta_{1}-\theta_{2}\right)^{3} \\
& \quad+A\left(\dot{\theta}_{1}-\dot{\theta}_{2}\right)=T_{1}, \\
& J_{2} \ddot{\theta}_{2}+c K(t)\left(\theta_{2}-\theta_{1}\right)+b K(t)\left(\theta_{2}-\theta_{1}\right)^{3} \\
& \quad+A\left(\dot{\theta}_{2}-\dot{\theta}_{1}\right)=T_{2},
\end{aligned}
$$

where $\ddot{\theta}_{1}, \ddot{\theta}_{2}$ are acceleration of rotational angle of moment of inertia. Combining (8) and (9), we have

$$
\begin{aligned}
& \left(\ddot{\theta}_{1}-\ddot{\theta}_{2}\right)+\frac{J_{1}+J_{2}}{J_{1} J_{2}} c K(t)\left(\theta_{1}-\theta_{2}\right) \\
& \quad+\frac{J_{1}+J_{2}}{J_{1} J_{2}} b K(t)\left(\theta_{1}-\theta_{2}\right)^{3}+\frac{J_{1}+J_{2}}{J_{1} J_{2}} A\left(\dot{\theta}_{1}-\dot{\theta}_{2}\right) \\
& =\frac{J_{2} T_{1}-J_{1} T_{2}}{J_{1} J_{2}} .
\end{aligned}
$$

Let

$$
\begin{aligned}
x & =\theta_{1}-\theta_{2}, \\
\dot{x} & =\dot{\theta}_{1}-\dot{\theta}_{2}, \\
\ddot{x} & =\ddot{\theta}_{1}-\dot{\theta}_{2}, \\
\frac{J_{2} T_{1}-J_{1} T_{2}}{J_{1} J_{2}} & =F \cos \left(\omega_{2} t\right), \\
a & =\frac{J_{1}+J_{2}}{J_{1} J_{2}}, \\
\lambda & =\frac{J_{1}+J_{2}}{J_{1} J_{2}} A .
\end{aligned}
$$

Hence, the above equation turns to

$$
\begin{aligned}
\ddot{x} & +a\left(k_{0}^{2}+k_{1} \cos \left(\omega_{1} t\right)\right)\left(c x+b x^{3}\right)+\lambda \dot{x} \\
& =F \cos \left(\omega_{2} t\right) .
\end{aligned}
$$

Equation (12) is dynamics equation of relative rotation system with Mathieu-Duffing oscillator.

\section{Melnikov's Method Analysis}

Melnikov's method [24-26] is an effective approach to predict chaotic behavior, its basic idea mainly makes the dynamics system into a Poincaré map system, and we obtain chaotic properties by studying conditions of the mapping system whether there exist homoclinic orbits or heteroclinic orbits.

Equation (12) can be transformed into first-order equation

$$
\begin{aligned}
\dot{x}_{1}= & x_{2}, \\
\dot{x}_{2}= & -a c k_{0}^{2} x_{1}-a b k_{0}^{2} x_{1}^{3}-a k_{1} \cos \left(\omega_{1} t\right)\left(c x_{1}+b x_{1}^{3}\right) \\
& -\lambda x_{2}+F \cos \left(\omega_{2} t\right) .
\end{aligned}
$$

Small parameter $\varepsilon$ is added to the nonlinear term of (13) and it may be written as

$$
\begin{aligned}
\dot{x}_{1} & =x_{2}, \\
\dot{x}_{2} & =-a c k_{0}^{2} x_{1}-a b k_{0}^{2} x_{1}^{3} \\
& -\varepsilon\left(a k_{1} \cos \left(\omega_{1} t\right)\left(c x_{1}+b x_{1}^{3}\right)+\lambda x_{2}\right. \\
& \left.-F \cos \left(\omega_{2} t\right)\right) .
\end{aligned}
$$

When $\varepsilon=0$, the above equation turns into

$$
\begin{aligned}
& \dot{x}_{1}=x_{2}, \\
& \dot{x}_{2}=-a c k_{0}^{2} x_{1}-a b k_{0}^{2} x_{1}^{3} .
\end{aligned}
$$

Equation (15) is a unperturbed Hamilton system and its Hamiltonian is

$$
H\left(x_{1}, x_{2}\right)=\frac{1}{2} x_{2}^{2}+\frac{1}{2} a c k_{0}^{2} x_{1}^{2}+\frac{1}{4} a b k_{0}^{2} x_{1}^{4} .
$$


There exist heteroclinic orbits and they satisfy

$$
\begin{gathered}
\dot{x}_{1}=x_{2}, \\
H\left( \pm \sqrt{-\frac{c}{b}}, 0\right)=\frac{1}{2} x_{2}^{2}+\frac{1}{2} a c k_{0}^{2} x_{1}^{2}+\frac{1}{4} a b k_{0}^{2} x_{1}^{4} .
\end{gathered}
$$

Then the parametric equations of two heteroclinic orbits are

$$
\begin{aligned}
& x_{1}(t)= \pm \sqrt{-\frac{c}{b}} \tanh \left(\frac{\sqrt{2 a c}}{a} k_{0} t\right), \\
& x_{2}(t)= \pm \frac{c k_{0}}{2} \sqrt{-\frac{2 a}{b}} \frac{1}{\cosh ^{2}}\left(\frac{\sqrt{2 a c}}{a} k_{0} t\right) .
\end{aligned}
$$

Melnikov function can be given by

$$
\begin{aligned}
& M\left(t_{0}\right)=\int_{-\infty}^{+\infty} x_{2}(t) \\
& \cdot\left[-a k_{1} \cos \left(\omega_{1}\left(t+t_{0}\right)\right)\left(c x_{1}(t)+b x_{1}(t)^{3}\right)\right. \\
& \left.-\lambda x_{2}(t)+F \cos \left(\omega_{2}\left(t+t_{0}\right)\right)\right] d t \\
& = \pm a c k_{1} \int_{-\infty}^{+\infty} x_{1}(t) x_{2}(t) \cos \left(\omega_{1}\left(t+t_{0}\right)\right) d t \\
& \pm a b k_{1} \int_{-\infty}^{+\infty} x_{1}(t)^{3} x_{2}(t) \cos \left(\omega_{1}\left(t+t_{0}\right)\right) d t \\
& -\lambda \int_{-\infty}^{+\infty} x_{2}(t)^{2} d t \pm F \int_{-\infty}^{+\infty} x_{2}(t) \cos \left(\omega_{2}\left(t+t_{0}\right)\right) d t
\end{aligned}
$$

where

$$
\begin{aligned}
& \int_{-\infty}^{+\infty} x_{1}(t) x_{2}(t) \cos \left(\omega_{1}\left(t+t_{0}\right)\right) d t \\
& =\int_{-\infty}^{+\infty} x_{1}(t) x_{2}(t) \sin \left(\omega_{1} t\right) d t \sin \left(\omega_{1} t_{0}\right) \\
& \int_{-\infty}^{+\infty} x_{1}(t)^{3} x_{2}(t) \cos \left(\omega_{1}\left(t+t_{0}\right)\right) d t \\
& =\int_{-\infty}^{+\infty} x_{1}(t)^{3} x_{2}(t) \sin \left(\omega_{1} t\right) d t \sin \left(\omega_{1} t_{0}\right) \\
& \int_{-\infty}^{+\infty} x_{2}(t) \cos \left(\omega_{2}\left(t+t_{0}\right)\right) d t \\
& =\int_{-\infty}^{+\infty} x_{2}(t) \cos \left(\omega_{2} t\right) d t \cos \left(\omega_{2} t_{0}\right)
\end{aligned}
$$

Let

$$
\begin{aligned}
& Z_{1}=\int_{-\infty}^{+\infty} x_{2}(t)^{2} d t, \\
& Z_{2}=\int_{-\infty}^{+\infty} x_{1}(t) x_{2}(t) \sin \left(\omega_{1} t\right) d t, \\
& Z_{3}=\int_{-\infty}^{+\infty} x_{1}(t)^{3} x_{2}(t) \sin \left(\omega_{1} t\right) d t \\
& Z_{4}=\int_{-\infty}^{+\infty} x_{2}(t) \cos \left(\omega_{2} t\right) d t .
\end{aligned}
$$

Substituting $Z_{1}-Z_{4}$ into Melnikov function, we have

$$
\begin{aligned}
M\left(t_{0}\right)= & -\lambda Z_{1} \pm\left(a c k_{1} Z_{2}+a b k_{1} Z_{3}\right) \sin \left(\omega_{1} t_{0}\right) \\
& \pm F Z_{4} \cos \left(\omega_{2} t\right) .
\end{aligned}
$$

When $\omega_{1}=\omega_{2}=\sqrt{a c k_{0}^{2}},(22)$ can be written as

$$
\begin{aligned}
& M\left(t_{0}\right) \\
& =-\lambda Z_{1} \\
& \quad \pm \sqrt{\left(a c k_{1} Z_{2}+a b k_{1} Z_{3}\right)^{2}+\left(F Z_{4}\right)^{2}} \sin \left(\omega_{1} t_{0}+\varphi\right),
\end{aligned}
$$

where

$$
\begin{aligned}
& \cos \varphi=\frac{a c k_{1} Z_{2}+a b k_{1} Z_{3}}{\sqrt{\left(a c k_{1} Z_{2}+a b k_{1} Z_{3}\right)^{2}+\left(F Z_{4}\right)^{2}}}, \\
& \sin \varphi=\frac{F Z_{4}}{\sqrt{\left(a c k_{1} Z_{2}+a b k_{1} Z_{3}\right)^{2}+\left(F Z_{4}\right)^{2}}} .
\end{aligned}
$$

Because of $M\left(t_{0}\right)=0$, we have

$$
\sin \left(\omega_{1} t_{0}+\varphi\right)= \pm \frac{\lambda Z_{1}}{\sqrt{\left(a c k_{1} Z_{2}+a b k_{1} Z_{3}\right)^{2}+\left(F Z_{4}\right)^{2}}}
$$

Due to $\left|\sin \left(\omega_{1} t_{0}+\varphi\right)\right| \leq 1$, then

$$
\begin{aligned}
& \left|\frac{\lambda Z_{1}}{\sqrt{\left(a c k_{1} Z_{2}+a b k_{1} Z_{3}\right)^{2}+\left(F Z_{4}\right)^{2}}}\right| \leq 1, \\
& \frac{\partial M\left(t_{0}\right)}{\partial t_{0}}= \pm \omega_{1} \sqrt{\left(a c k_{1} Z_{2}+a b k_{1} Z_{3}\right)^{2}+\left(F Z_{4}\right)^{2}} \\
& \cdot \cos \left(\omega_{1} t_{0}+\varphi\right) \neq 0 .
\end{aligned}
$$

That is, $\cos \left(\omega_{1} t_{0}+\varphi\right) \neq 0$, so $\sin \left(\omega_{1} t_{0}+\varphi\right) \neq 0$; we can know

$$
\left|\frac{\lambda Z_{1}}{\sqrt{\left(a c k_{1} Z_{2}+a b k_{1} Z_{3}\right)^{2}+\left(F Z_{4}\right)^{2}}}\right|<1 .
$$

There exists a sufficient small $\varepsilon$, making $M\left(t_{0}\right)=0$ and $\partial M\left(t_{0}\right) / \partial t_{0} \neq 0$; therefore, the system produces chaotic behavior.

For system (13), taking parameters $a c k_{0}^{2}=4, a b k_{0}^{2}=-4$, $a c k_{1}=1, a b k_{1}=-1, \lambda=0.5, F=1.42$, and $\omega_{1}=\omega_{2}=2$ with the initial conditions $x(0)=1, \dot{x}(0)=0$, the Poincaré map and phase portraits were plotted by using the numerical simulation in Figure 1.

From Figure 1, we can see that the Poincaré map has chaotic attractors, phase portraits show nonoverlapping and are disorganized, and these can prove that relative rotation system with Mathieu-Duffing oscillator is chaos. 


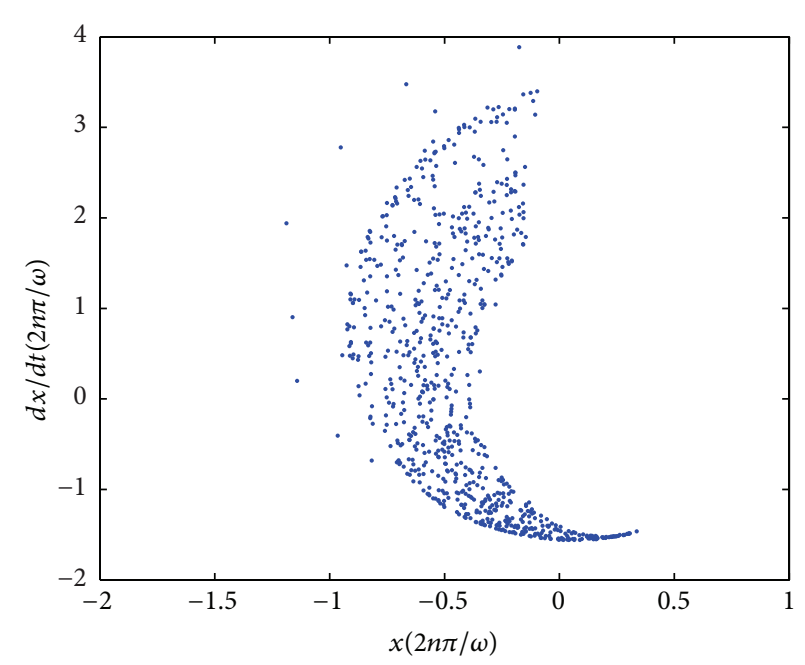

(a)

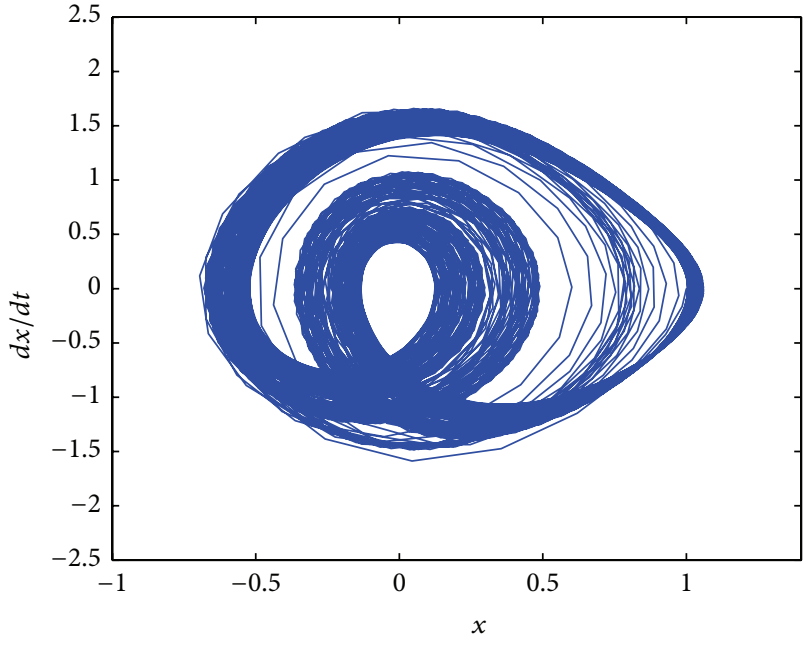

(b)

FIgURE 1: (a) Poincaré map; (b) phase portraits.

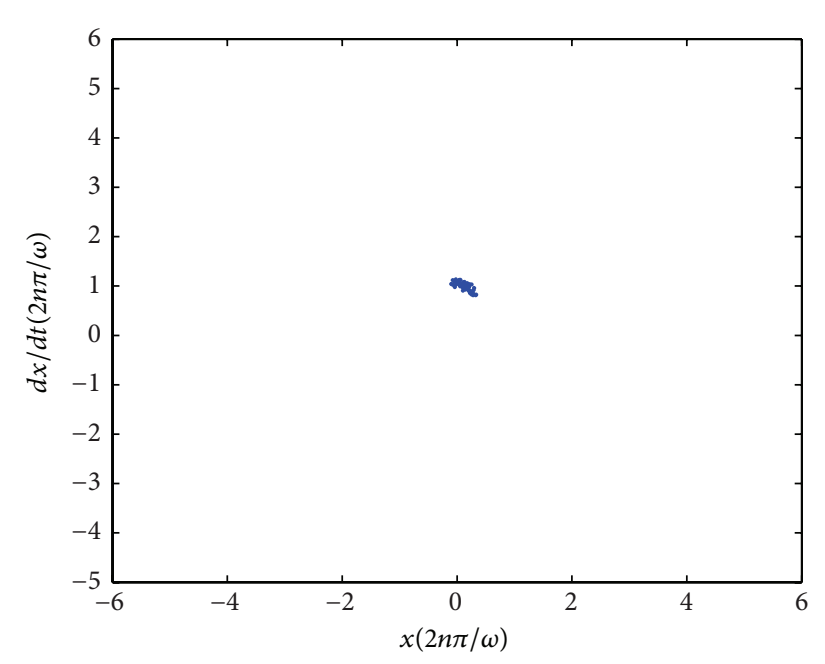

(a)

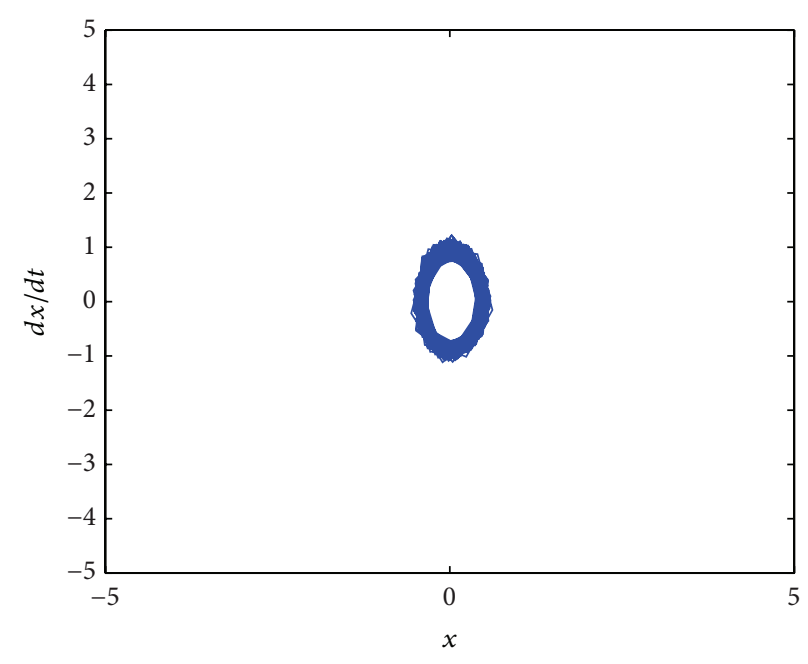

(b)

Figure 2: (a) Poincaré map with $\sigma=0.4$; (b) phase portraits with $\sigma=0.4$.

\section{Chaos Control of Relative}

\section{Rotation System with Mathieu-Duffing} Oscillator Using Noise

Adding Gaussian white noise, (13) can be written as

$$
\begin{aligned}
\dot{x}_{1}= & x_{2}, \\
\dot{x}_{2}= & -a c k_{0}^{2} x_{1}-a b k_{0}^{2} x_{1}^{3}-a k_{1} \cos \left(\omega_{1} t\right)\left(c x_{1}+b x_{1}^{3}\right) \\
& -\lambda x_{2}+F \cos \left(\omega_{2} t+\sigma \xi(t)\right),
\end{aligned}
$$

where $\xi(t)$ denotes standard Gaussian white noise; it satisfies

$$
\begin{aligned}
E \xi(t) & =0, \\
E \xi(t) \xi(t+\tau) & =\zeta(\tau),
\end{aligned}
$$

where $\zeta(\tau)$ is Dirac-Delta function and $\sigma$ is the intensity of noise.

Taking the same parameters and initial conditions as Section 3. Equation (28) can be written:

$$
\begin{aligned}
\dot{x}_{1}= & x_{2}, \\
\dot{x}_{2}= & -4 x_{1}+4 x_{1}^{3}-\cos (2 t) x_{1}+\cos (2 t) x_{1}^{3}-0.5 x_{2} \\
& +1.42 \cos (2 t+\sigma \xi(t)) .
\end{aligned}
$$

Making the Poincaré map as

$\Sigma \longrightarrow \Sigma$,

$$
\Sigma\left\{(x(t), \dot{x}(t)) \mid t=0, \frac{2 \pi}{\omega_{2}}, \frac{4 \pi}{\omega_{2}}, \ldots\right\} \subseteq \mathbb{R}^{2} .
$$


Under the given initial conditions, the solution of differential equation (30) is solved by the fourth-order Runge-Kutta method and the solution is plotted for every $T=2 \pi / \omega_{2}$. After deleting the first 100 dots, we plot the Poincaré map by using surplus 200 iteration dots, when the intensity of noise $\sigma=0.4$; it is shown in Figure 2(a).

The Poincaré map appears to be a stable stochastic attractor, which means that the system is stable. To further verify the obtained results, we plotted the phase portraits. Taking the same intensity of noise, the phase portraits are shown in Figure 2(b). We can see from Figure 2(b) that the phase portraits turned into a regular annulus. It is proved that chaotic behavior was suppressed, and the system changed chaos state into stable state.

\section{Concluding Remarks}

We investigate the chaos analysis and control of relative rotation system with Mathieu-Duffing oscillator in this paper. By analysis of Melnikov's method, we confirm chaotic behavior of this system under the given parameters and initial conditions. Adding the Gaussian white noise to phase of system, we plot the Poincaré map and phase portraits under the intensity of noise $\sigma=0.4$. We get that the Poincaré map changed chaotic attractors into a stable attractor, and phase portraits also displayed regular state, so the relative rotation system with Mathieu-Duffing oscillator eventually becomes stable.

\section{Conflict of Interests}

The authors declare that there is no conflict of interests regarding the publication of this paper.

\section{References}

[1] B. L. Hao, Elementary Symbolic Dynamics and Chaos in Dissipative Systems, World Scientific Publishing, Singapore, 1989.

[2] Z. T. Zhusubaliyev and E. Mosekilde, Bifurcations and Chaos in Piecewise-Smooth Dynamical Systems, vol. 44 of World Scientific Series on Nonlinear Science. Series A: Monographs and Treatises, World Scientific, River Edge, NJ, USA, 2003.

[3] G. S. Chen and J.-Y. Chang, "Chaos in nonlinear dynamics of air bearing slider in contact," Microsystem Technologies, vol. 20, no. 8-9, pp. 1739-1744, 2014.

[4] L. Chen and G. Chen, "Controlling chaos in an economic model," Physica A, vol. 374, no. 1, pp. 349-358, 2007.

[5] B. Mensour and A. Longtin, "Chaos control in multistable delay-differential equations and their singular limit maps," Physical Review E-Statistical Physics, Plasmas, Fluids, and Related Interdisciplinary Topics, vol. 58, no. 1, pp. 410-422, 1998.

[6] S. Yanchuk, M. Wolfrum, P. Hovel, and E. Scholl, "Control of unstable steady states by long delay feedback," Physical Review E, vol. 74, Article ID 026201, 2006.

[7] Y. Lei, W. Xu, Y. Xu, and T. Fang, "Chaos control by harmonic excitation with proper random phase," Chaos, Solitons \& Fractals, vol. 21, no. 5, pp. 1175-1181, 2004.

[8] Y. F. Gu and J. Xiao, "Analysis of the Willis chaotic system and the control of random phase," Acta Physica Sinica, vol. 63, pp. $1-7,2014$.
[9] X. Cong and L. Li, "Suppressing chaos of Warship power system based on the quantum mechanics theory," International Journal of Theoretical Physics, vol. 53, no. 8, pp. 2778-2784, 2014.

[10] L. Jin, J. Mei, and L. Li, "Chaos control of parametric driven Duffing oscillators," Applied Physics Letters, vol. 104, Article ID 134101, 2014.

[11] R. Lima and M. Pettini, "Suppression of chaos by resonant parametric perturbations," Physical Review A, vol. 41, no. 2, pp. 726-733, 1990 .

[12] L. S. Jin, J. Mei, and L. Li, "Chaos control of parametric driven Duffing oscillators," Applied Physics Letters, vol. 104, Article ID 134101, 6 pages, 2014.

[13] P. Hövel and E. Schöll, "Control of unstable steady states by time-delayed feedback methods," Physical Review E, vol. 72, no. 4, Article ID 046203, pp. 23-46, 2005.

[14] Y.-Z. Wang and F.-M. Li, "Dynamical properties of Duffingvan der Pol oscillator subject to both external and parametric excitations with time delayed feedback control," Journal of Vibration and Control, vol. 21, no. 2, pp. 371-387, 2015.

[15] M. Carmeli, "Rotational relativity theory," International Journal of Theoretical Physics, vol. 25, no. 1, pp. 89-94, 1986.

[16] P.-M. Shi, D.-Y. Han, and B. Liu, "Chaos and chaotic control in a relative rotation nonlinear dynamical system under parametric excitation," Chinese Physics B, vol. 19, no. 9, Article ID 090306, 2010.

[17] S. Liu, X. Li, Y. Li, and H. Li, "Stability and bifurcation for a coupled nonlinear relative rotation system with multi-time delay feedbacks," Nonlinear Dynamics, vol. 77, no. 3, pp. 923934, 2014.

[18] M. Ramesh and S. Narayanan, "Chaos control by nonfeedback methods in the presence of noise," Chaos, Solitons and Fractals, vol. 10, no. 9, pp. 1473-1489, 1999.

[19] W. Y. Liu, W. Q. Zhu, and Z. L. Huang, "Effect of bounded noise on chaotic motion of a Duffing oscillator under parametric excitation," Chaos, solitons \& fractals, vol. 12, no. 3, pp. 527-537, 2001.

[20] C. L. Wu, Y. Lei, and T. Fang, "Stochastic chaos in a Duffing oscillator and its control," Chaos, Solitons and Fractals, vol. 27, no. 2, pp. 459-469, 2006.

[21] J. L. Yin, Q. Q. Xing, and L. X. Tian, "Melnikov analysis and chaos control of nonlinear dispersive $\mathrm{KdV}$ equation under external periodic perturbation," Indian Journal of Physics, vol. 89, no. 3, pp. 273-279, 2015.

[22] D.-X. Hou, H.-X. Zhao, and B. Liu, "Bifurcation and chaos in some relative rotation systems with Mathieu-Duffing oscillator," Acta Physica Sinica, vol. 62, no. 23, Article ID 234501, 2013.

[23] B. Liu, H. X. Zhao, and D. X. Hou, "Bifurcation and chaos of some relative rotation system with triple-well Mathieu-Duffing oscillator," Acta Physica Sinica, vol. 17, pp. 1-9, 2014.

[24] J. L. Yin, L. W. Zhao, and L. Y. Tian, "Melnikovs criteria and chaos analysis in the nonlinear Schrödinger equation with Kerr law nonlinearity," Abstract and Aapplied Analysis, vol. 10, pp. 1$12,2014$.

[25] L. Cveticanin and M. Zukovic, "Melnikov's criteria and chaos in systems with fractional order deflection," Journal of Sound and Vibration, vol. 326, no. 3-5, pp. 768-779, 2009.

[26] S. Li, Q. Li, J. R. Li, and J. Q. Feng, "Chaos prediction and control of Goodwin's nonlinear accelerator model," Nonlinear Analysis: Real World Applications, vol. 12, no. 4, pp. 1950-1960, 2011. 


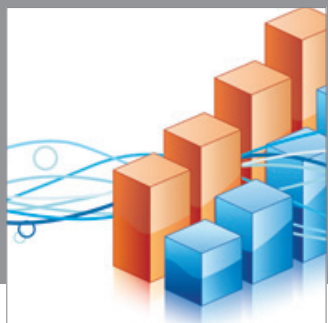

Advances in

Operations Research

mansans

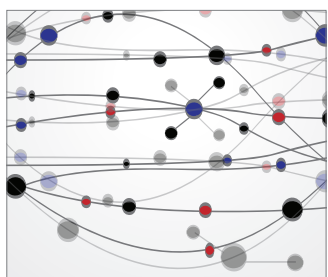

The Scientific World Journal
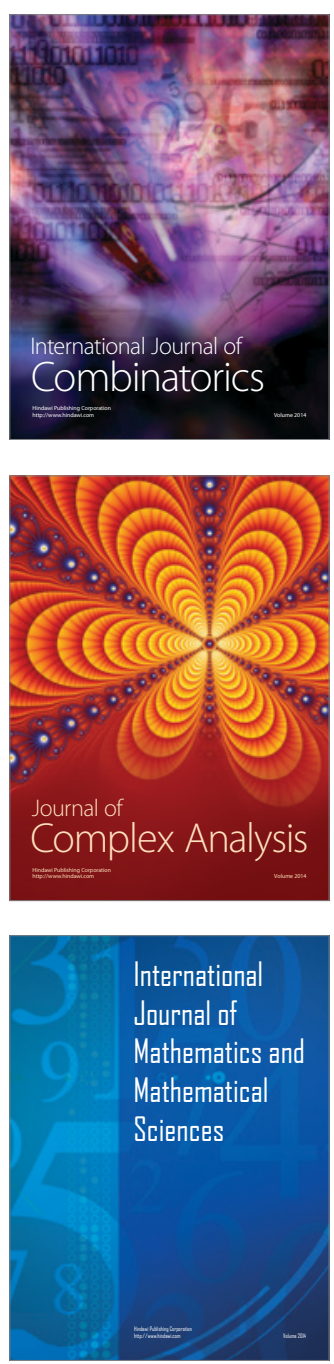
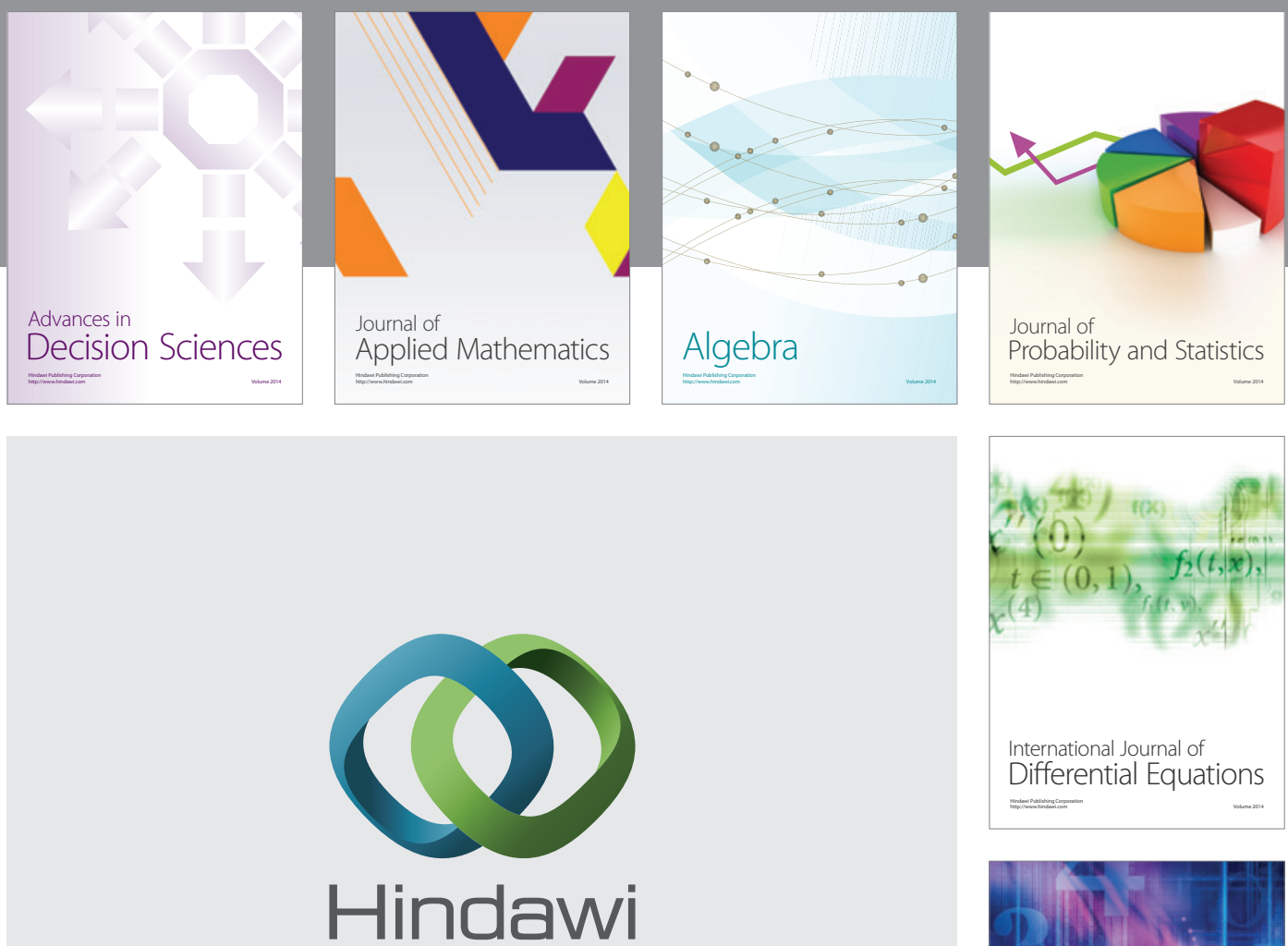

Submit your manuscripts at http://www.hindawi.com
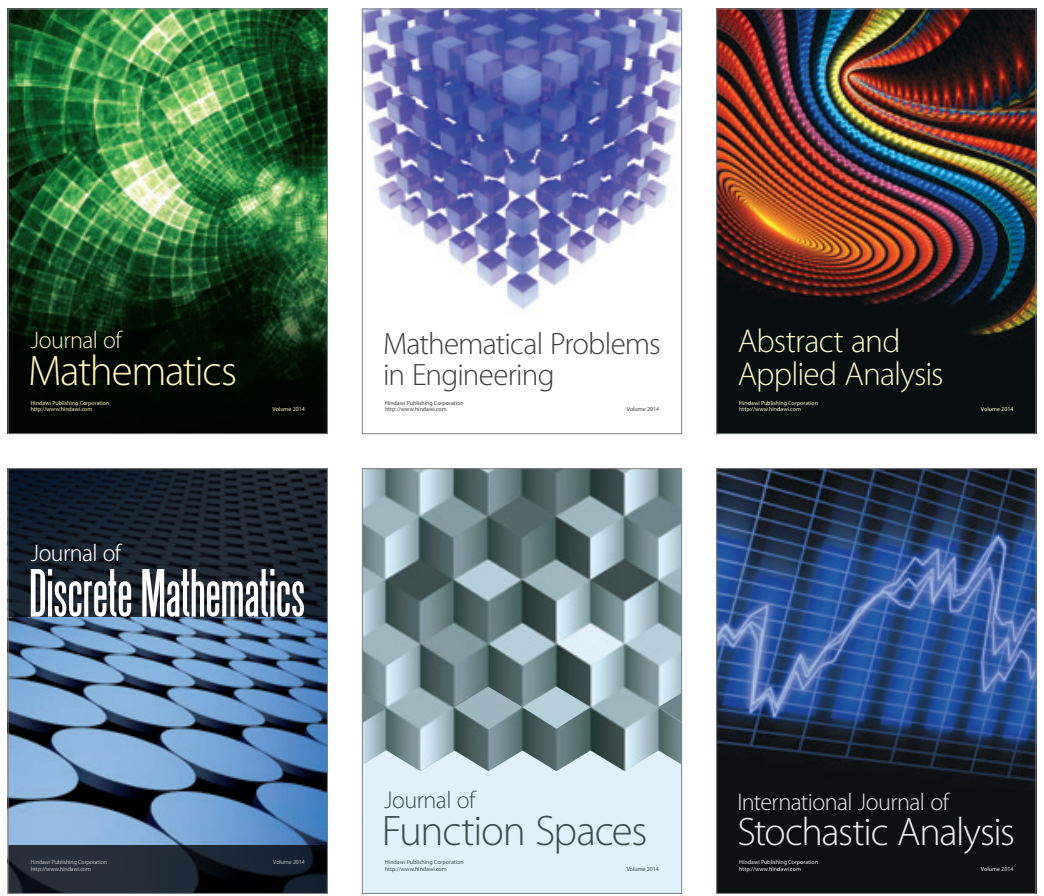

Journal of

Function Spaces

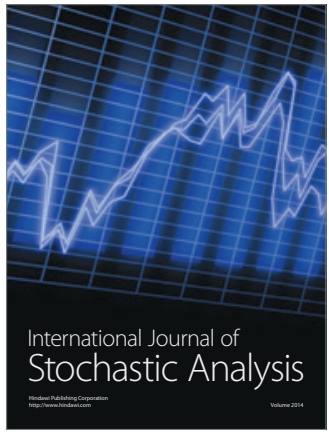

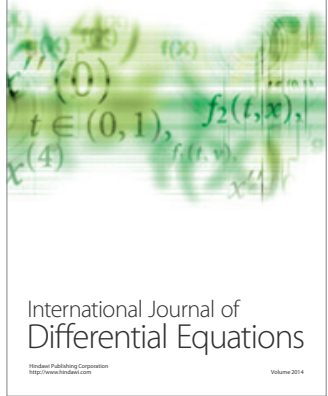
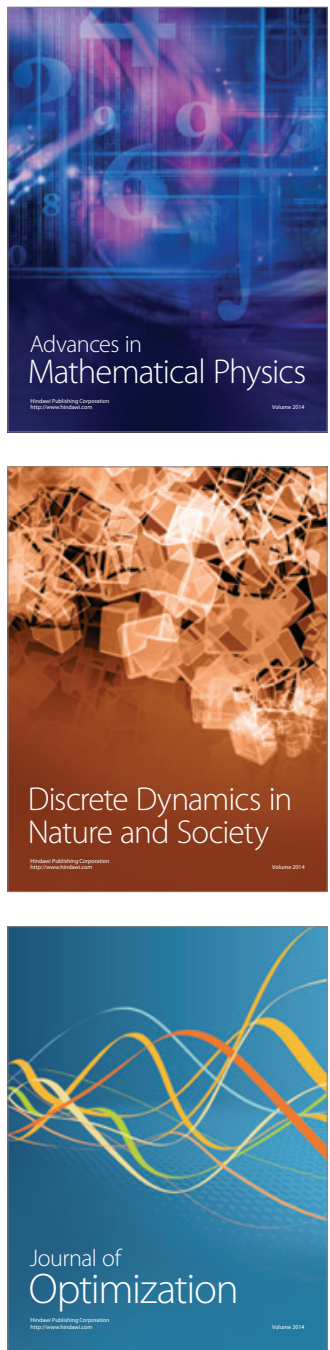